\title{
El cariotipo de Chaetanthera renifolia (J. Remy) Cabrera (Asteraceae)
}

\section{The karyotype of Chaethanthera renifolia (J. Remy) Cabrera (Asteraceae)}

\author{
Carlos M. Baeza, Eduardo Ruiz \& Cristian Torres-Díaz \\ Departamento de Botánica, Universidad de Concepción, Casilla 160-C, Concepción-Chile. \\ cbaeza@udec.cl
}

\begin{abstract}
The karyotype of Chaetanthera renifolia (J. Remy) Cabrera from Chile was examined. The species has $2 \mathrm{n}=2 \mathrm{x}=44$ chromosomes, with $1 \mathrm{~m}+1 \mathrm{st}+2 \mathrm{st}$-sat $+17 \mathrm{t}+1 \mathrm{t}$-sat chromosomes. The reported karyotype is bimodal and very asymmetric $($ AsK $\%=86.6$, TF $\%=13.4$ and Syi $\%=15.5)$. These results are indicating that this species has the highest known chromosome number of the genus so far and the most asymmetrical karyotype of the group.
\end{abstract}

El género Chaetanthera Ruiz et Pav. (Asteraceae: Mutisieae) es endémico del sur de Sudamérica y está compuesto de 42 especies, de las cuales 36 se encuentran en Chile, la mayoría concentradas en la zona de clima mediterráneo y de desierto con lluvias de invierno (Cabrera 1937). En la actualidad, sólo existe información cariológica detallada en algunas de las especies de los subgéneros Euchaetanthera, Egania y Proselia, esto es, características específicas del cariotipo, además del número cromosómico. Chaetanthera renifolia (J.Remy) Cabrera es una especie perenne, endémica de los Andes de la Región Metropolitana de Chile, donde crece en el piso altoandino ocupando de preferencia las laderas y planicies arenosas o pedregosas (Riedemann et al. 2008). En este trabajo se entrega una descripción detallada del cariotipo de esta especie que pertenece al subgénero Tylloma, hasta ahora no analizado citológicamente.

Se estudió citológicamente una población de Chaetanthera renifolia recolectada en la Región Metropolitana, Provincia de Santiago, Comuna de Lo Barnechea, Centro de Sky La Parva, Pista Los Barros,

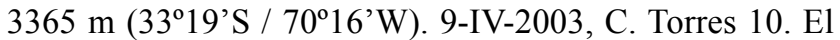
material de referencia está depositado en el Herbario de la Universidad de Concepción (CONC).

Se analizaron 15 placas metafásicas provenientes de 10 semillas germinadas y los cromosomas se midieron siguiendo la metodología propuesta por Baeza et al. (2006). Para la población analizada se determinó el índice de asimetría del cariotipo (AsK \%) definido por Arano y Saito (1980), el TF\% = índice de asimetría de Huziwara (1962) el Syi = índice de asimetría de Venora et al. (2002), el cociente entre el par de cromosomas más largo y el más corto $(\mathrm{R})$ y la longitud total del genoma (TCL, expresada en $\mu \mathrm{m}$ ). Los cromosomas fueron clasificados de acuerdo a Levan et al. (1964).

Chaetanthera renifolia presenta $2 \mathrm{n}=2 \mathrm{x}=44$ cromosomas (Fig. 1A y B), con un cariotipo bimodal muy asimétrico con un complemento cromosómico haploide de $1 \mathrm{~m}+1 \mathrm{st}+2 \mathrm{st}-$ sat $+17 \mathrm{t}+1 \mathrm{t}$-sat (Tabla I), esto es, 1 par de cromosomas metacéntricos, tres pares subtelocéntricos, dos de ellos con satélites y 18 pares telocéntricos, uno de ellos con satélite. El cociente entre el par más largo y el par más corto $(\mathrm{R})$ fue 3,5. El índice de asimetría del cariotipo (AsK\%) fue de 86,6 , el TF\% 13,4, el Syi 15,5, lo que está indicando el alto grado de asimetría del cariotipo. La longitud total del genoma (LTC) fue de 170,4 $\pm 8,2 \mu \mathrm{m}$. La longitud de los cromosomas fluctúa entre 7-2 $\mu \mathrm{m}$ (Tabla I).

Las características cariotípicas de Chaetanthera renifolia resultaron ser excepcionalmente particulares, dado el gran número de cromosomas y su extraordinaria asimetría. Por un lado, el número máximo de cromosomas reportados para el género es de 28 para $C$. glabrata (Grau 1987) y para $C$. tenella (Powell et al. 1974), sin embargo, en C. renifolia el 2 n casi duplica este número.

A pesar de que no se encontraron los dos pares de homólogos de cada uno de los cromosomas, no se descarta la posibilidad de que se trate de un alopoliploide, puesto que existen otras especies con $2 \mathrm{n}=22$ (Baeza et al. 2009). Además, esta especie crece en una zona de altura y en un clima frío, donde la poliploidía podría llegar a darse con mayor probabilidad asegurando el éxito reproductivo de un híbrido. 


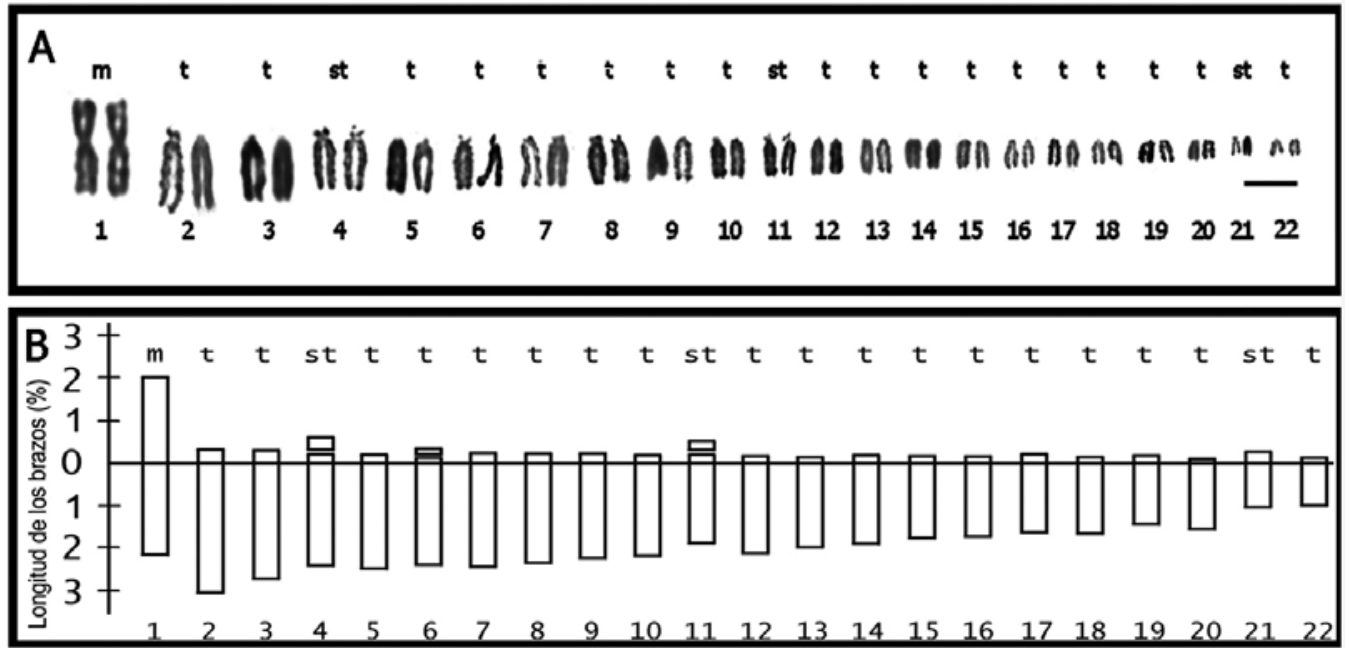

Figura I. A. Cariotipo bimodal de Chaetanthera renifolia (J.Remy) Cabrera $(2 n=44)$, obtenido del análisis de 15 placas metafásicas. B. Idiograma del complemento haploide (los cromosomas se han ordenado de acuerdo a su tamaño decreciente). Escala $=$ a $5 \mu \mathrm{m}$.

Figure I. A. Karyotype bimodal of Chaetanthera renifolia (J.Remy) Cabrera $(2 n=44)$, using 15 metaphasic plates B. Ideogram of the haploid chromosomes complement (the chromosomes have been ordered according to decreasing size). Scale $=5 \mu \mathrm{m}$.

TABLA I. Longitud promedio de los cromosomas de Chaetanthera renifolia (J.Remy) Cabrera calculados en porcentaje de la longitud del genoma haploide total de 15 metafases.

TABLE I. Average length of chromosomes of Chaetanthera renifolia (J.Remy) Cabrera calculated in percent of the mean haploid genome length of 15 metaphases.

\begin{tabular}{|c|c|c|c|c|c|c|}
\hline $\begin{array}{c}\text { Par } \\
\text { cromosómico }\end{array}$ & $\begin{array}{c}\text { Brazo largo } \\
(\%) \pm \text { D.S. }\end{array}$ & $\begin{array}{c}\text { Brazo corto } \\
(\%) \pm \text { D.S. }\end{array}$ & $\begin{array}{c}\text { Largo } \\
\text { relativo }(\%)\end{array}$ & $\begin{array}{l}\text { Largo total } \\
\qquad(\mu \mathrm{m})\end{array}$ & $\begin{array}{l}\text { Radio del brazo } \\
\text { (L/C) }\end{array}$ & $\begin{array}{c}\text { Tipo de } \\
\text { cromosoma }\end{array}$ \\
\hline 1 & $2,13 \pm 0,10$ & $2,01 \pm 0,08$ & 4,14 & 7,0 & 1,06 & $\mathrm{~m}$ \\
\hline 2 & $3,02 \pm 0,12$ & $0,31 \pm 0,10$ & 3,33 & 6,0 & 9,7 & $\mathrm{t}$ \\
\hline 3 & $2,72 \pm 0,10$ & $0,29 \pm 0,12$ & 3,01 & 5,1 & 9,4 & $\mathrm{t}$ \\
\hline 4 & $2,41 \pm 0,12$ & $0,51 \pm 0,14$ & 2,92 & 4,9 & 4,7 & st-sat \\
\hline 5 & $2,46 \pm 0,14$ & $0,19 \pm 0,14$ & 2,65 & 4,5 & 13,0 & $\mathrm{t}$ \\
\hline 6 & $2,31 \pm 0,12$ & $0,32 \pm 0,10$ & 2,63 & 4,4 & 7,2 & t-sat \\
\hline 7 & $2,40 \pm 0,10$ & $0,25 \pm 0,12$ & 2,60 & 4,4 & 9,6 & $\mathrm{t}$ \\
\hline 8 & $2,23 \pm 0,14$ & $0,25 \pm 0,16$ & 2,48 & 4,2 & 8,9 & $\mathrm{t}$ \\
\hline 9 & $2,21 \pm 0,12$ & $0,22 \pm 0,08$ & 2,43 & 4,1 & 10,0 & $\mathrm{t}$ \\
\hline 10 & $2,15 \pm 0,10$ & $0,18 \pm 0,10$ & 2,33 & 3,9 & 11,9 & $\mathrm{t}$ \\
\hline 11 & $1,86 \pm 0,10$ & $0,41 \pm 0,12$ & 2,27 & 3,8 & 4,5 & st-sat \\
\hline 12 & $2,10 \pm 0,12$ & $0,16 \pm 0,10$ & 2,26 & 3,8 & 13,1 & $\mathrm{t}$ \\
\hline 13 & $1,96 \pm 0,10$ & $0,14 \pm 0,10$ & 2,10 & 3,6 & 14,0 & $\mathrm{t}$ \\
\hline 14 & $1,88 \pm 0,12$ & $0,18 \pm 0,10$ & 2,06 & 3,5 & 10,4 & $\mathrm{t}$ \\
\hline 15 & $1,74 \pm 0,10$ & $0,17 \pm 0,12$ & 1,91 & 3,2 & 10,2 & $\mathrm{t}$ \\
\hline 16 & $1,71 \pm 0,10$ & $0,15 \pm 0,10$ & 1,86 & 3,2 & 11,4 & $\mathrm{t}$ \\
\hline 17 & $1,62 \pm 0,13$ & $0,22 \pm 0,12$ & 1,84 & 3,1 & 7,4 & $\mathrm{t}$ \\
\hline 18 & $1,64 \pm 0,12$ & $0,14 \pm 0,10$ & 1,78 & 3,0 & 11,7 & $\mathrm{t}$ \\
\hline 19 & $1,42 \pm 0,10$ & $0,17 \pm 0,10$ & 1,58 & 2,7 & 8,4 & $\mathrm{t}$ \\
\hline 20 & $1,54 \pm 0,15$ & $0,10 \pm 0,12$ & 1,55 & 2,6 & 15,4 & $\mathrm{t}$ \\
\hline 21 & $1,03 \pm 0,12$ & $0,26 \pm 0,12$ & 1,29 & 2,2 & 4,0 & st \\
\hline 22 & $0,98 \pm 0,10$ & $0,12 \pm 0,10$ & 1,10 & 2,0 & 8,2 & $\mathrm{t}$ \\
\hline
\end{tabular}


Por otra parte, C. renifolia presenta un cariotipo muy asimétrico con un solo par de cromosomas metacéntricos y 21 pares acrocéntricos. El cariotipo más asimétrico reportado hasta la fecha es el de C. microphylla que presenta 8 pares de cromosomas metacéntricos y 4 pares acrocéntricos (Baeza \& Schrader 2005). Esta peculiaridad de C. renifolia también ha quedado de manifiesto en el estudio evolutivo realizado por Hershkovitz et al. (2006), en donde esta especie es la única del subgénero Tylloma que está más relacionada a especies de otros subgéneros (Carmelita y Euchaetanthera) que con especies de su propio subgénero. Además, la divergencia de C. renifolia parece haber sido bastante temprana dentro del género.

Por otro lado, los resultados cariológicos son congruentes con las tendencias evolutivas del cariotipo para el género, propuestas por Baeza et al. (2009), donde se señala que, en general, las especies de divergencia temprana tendrían mayor número de cromosomas que aquéllas de divergencia más reciente, y que las especies que crecen a mayor altura tienden a tener cariotipos más asimétricos. De ahí entonces la importancia de ampliar los estudios a aquellas especies que crecen a mayor altitud en la Cordillera de los Andes y también a aquellas especies que, según Hershkovitz et al. (2006), habrían tenido una divergencia más reciente.

\section{AGRADECIMIENTOS}

Se agradece el apoyo de Fondecyt $\mathrm{N}^{\circ} 1070520$ y al Departamento de Botánica de la Universidad de Concepción por las facilidades otorgadas para realizar esta investigación. Cristian Torres-Díaz agradece al proyecto Fondecyt postdoctoral 3090020.

\section{BIBLIOGRAFÍA}

Arano, H. \& H. Saito. 1980. Cytological studies in family Umbelliferae 5. Karyotypes of seven species in subtribe Seselinae. La Kromosomo 2: 471-480.

Baeza, C. \& O. Schrader. 2005. Análisis del cariotipo y detección de los genes $5 \mathrm{~S}$ y $18 \mathrm{~S} / 25 \mathrm{~S}$ rDNA en Chaetanthera microphylla (Cass.) H. et A. (Asteraceae). Gayana Botánica 62(1): 49-51.

Baeza, M., O. Schrader, E. Ruiz \& M. Negritto. 2006. Análisis comparativo del cariotipo en poblaciones de Alstroemeria ligtu subsp. ligtu y A. ligtu subsp. simsii (Alstroemeriaceae) de Chile. Darwiniana 44(2): 313-318.

Baeza, M., E. Ruiz \& M. Negritto. 2009. Importancia del cariotipo en la taxonomía y evolución del género Chaetanthera (Asteraceae): evidencias preliminares para especies que crecen en Chile. Gayana Botánica 66(1): 50-57.

CABRERA,A. 1937. Revisión del género Chaetanthera (Compositae). Revista del Museo de La Plata Sección Botánica 1: 87215.

GraU, J. 1987. Chromosomenzahlen chilenischer Mutisieen (Compositae). Botanische Jahrbücher 108: 229-237.

Hershkovitz, M., M.T.K. Arroyo, C. Bell \& L. Hinojosa. 2006. Phylogeny of Chaetanthera (Asteraceae: Mutisieae) reveals both ancient and recent origins of the high elevation lineages. Molecular Phylogenetics and Evolution 41: 594605.

Huziwara, Y. 1962. Karyotype analysis in some genera of Compositae. VIII. Further studies on the chromosomes of Aster. American Journal of Botany 49: 116-119.

Levan, A., K. Fredga \& A. SAndberg. 1964. Nomenclature for centromeric position on chromosomes. Hereditas 52: 201220.

Powell, A., D. Kyhos \& P. Raven. 1974. Chromosome numbers in Compositae. X. American Journal of Botany 61: 909-913.

Riedemann, P., G. Aldunate \& S. Teillier. 2008. Flora nativa de valor ornamental. Zona Cordillerana de los Andes Identificación y propagación. Salesianos Impresores S.A. $674 \mathrm{pp}$.

Venora, G., S. Blangiforti, M. Ruffini Castiglioni, D. Pignone, F. Losavio \& R. CRemonini. 2002. Chromatin organisation and computer arded karyotyping of Triticum durum Desf. cv Timilia. Caryologia 55: 91-98.

Recibido: 28.04 .10

Aceptado: 23.06 .10 